\title{
Bearing Fault Diagnosis Based on IFA-ELM
}

\author{
Qunxian Chen and Zekun Zhou* \\ ${ }^{1}$ Shanghai Dianji University, Shanghai, China \\ ${ }^{*}$ Corresponding author
}

\begin{abstract}
Extreme learning machine (ELM) is a simple and effective feedforward neural network. It can be used in pattern recognition. But its classification ability is not good enough. In order to solve this problem, this paper proposed an improved firefly algorithm and used it in the parameters selection of ELM. After establishing the IFA-ELM model, we use UCI standard data set to verify its classification ability. Finally, the model is used in bearing fault diagnosis and obtains a good result.
\end{abstract}

Keywords-firefly algorithm, extreme learning machine, pattern recognition, fault diagnosis

\section{INTRODUCTION}

Basic neural network has the disadvantages about slow training speed and low accuracy. In order to solve this problem, researchers have put forward the extreme learning machine theory. As a single hidden layer feedforward neural network, extreme learning machine has lots of advantages. Compare with basic neural network, ELM is more simple and effective. ELM can randomly generate the connection weight ' $w$ ' between input layer and hidden layer and the hidden layer neuron threshold ' $b$ ' [1]. In addition, for these two randomly selected parameters, there is no need to adjust them in training process. By setting the number of neurons in the hidden layer, we can get the only optimal solution. ELM has the advantages about fast learning speed, good generalization performance, and so on [2].

However, when we use ELM to solve the pattern recognition problems, its classification ability is not good enough. In order to solve this problem, this paper proposed an improved firefly algorithm and use it in parameters selection of ELM.

The firefly algorithm is a kind of swarm stochastic optimization algorithm which is based on the characteristics of the light emitting and aggregation behaviors of the firefly in the natural world [3].

In the running process, firefly use their own fluorescein to forage food and communicate with other fireflies around. In general, the higher the individual fluorescein, the more fireflies will gather around. Firefly move through searching other individual, which is more bright [4].

But the basic firefly algorithm has the disadvantage about falling into local optimal solution. In order to solve this problem, this paper proposed an improved firefly algorithm by changing the displacement formula. The improved formula took the population information into account and the experimental results showed that the improved firefly algorithm can solve the premature problem and can be used in function optimization.
The parameters ' $w$ ' and ' $b$ ' are selected randomly in basic ELM [5]. In order to improve ELM's classification ability, the IFA is used to select these two parameters intelligently. After establishing the IFA-ELM model, UCI standard data set is used to verify its classification accuracy. Then, the model is used in bearing fault diagnosis work.

\section{IFA INTRODUCTION}

\section{A. Basic Firefly Algorithm}

In firefly algorithm, each firefly represents a potential solution of the problem [6]. In the iteration process, fireflies will continue to search around the more bright individual to update their own position and find better solution. Finally, fireflies will gather around the most bright individual to search the global optimal solution [7].

1) The fluorescence intensity of firefly can be defined as follows:

$$
I=I_{0} e^{-\gamma_{i j}}
$$

In the above formula, $I_{0}$ is the fluorescence intensity of firefly's current position; $\gamma$ is absorption coefficient; $r_{i j}$ is the spatial distance between the ith firefly and the jth firefly.

2) The attraction of firefly can be defined as follows:

$$
\beta=\beta_{0} e^{-r_{i j}^{2}}
$$

In the above formula, is the maximum fitness factor.

3) The displacement of firefly can be defined as follows:

$$
x_{i}^{k+1}=x_{i}^{k}+\beta\left(x_{j}-x_{i}\right)+\alpha\left(\text { rand }-\frac{1}{2}\right)
$$

In the above formula, $x_{i}$ and $x_{j}$ is the ith and jth firefly's position and the jth firefly is more bright than ith firefly. $\alpha$ is step length factor; $\alpha$ (rand $\left.-\frac{1}{2}\right)$ is the random disturbance term.

\section{B. Improved Firefly Algorithm}

In order to solve the problem that basic firefly algorithm is easy to fall into local optimal solution, we proposed IFA model. In this model, we put the most bright individual into account. So, fireflies can learn the best individual position in the process 
of moving. This method can increase the global search speed. In addition, in order to make fireflies move reasonably, two weight factors were set up to control them. On the other hand, we improve the fixed step length factor. The new step length factor will decrease linearly based on iteration number. This approach can make fireflies have higher global search ability in the initial iteration and have higher local search ability in later iteration.

The improved formula shows as follows:

$$
x_{i}^{k+1}=x_{i}^{k}+w_{1}\left(x_{\text {best }}-x_{i}\right)+w_{2} \beta\left(x_{j}-x_{i}\right)+\alpha\left(\text { rand }-\frac{1}{2}\right)
$$

In the above formula, $x_{\text {best }}$ is the best firefly's position.

$$
\begin{gathered}
w_{1}=0.4+0.5\left(\left(\exp \left(10\left(T_{\max }-t+1\right) /\left(T_{\max }-1\right)\right)-1\right) /(\exp (10)-1)\right)(5) \\
w_{2}=0.4+0.5\left(\left(\exp \left(10(t-1) /\left(T_{\max }-1\right)\right)-1\right) /(\exp (10)-1)\right) \\
\alpha=0.2+0.6\left(T_{\max }-t\right) / T_{\max }
\end{gathered}
$$

In the above formula, $T_{\max }$ is the maximum number of iteration; $t$ is the current iteration number.

\section{IFA-ELM MODEL}

ELM is composed of three parts, input layer, hidden layer and output layer. These three layers are connected by neurons [8]. There are $\mathrm{n}$ neurons in the input layer, $l$ neurons in the hidden layer, $m$ neurons in the output layer. The connection weight between the input layer and the hidden layer and the connection weight between the hidden layer and output layer can be defined as follows:

$$
w=\left[\begin{array}{cccc}
\omega_{11} & \omega_{12} & \cdots & \omega_{1 n} \\
\omega_{21} & \omega_{22} & \cdots & \omega_{2 n} \\
\vdots & \vdots & & \vdots \\
\omega_{l 1} & \omega_{l 2} & \cdots & \omega_{l n}
\end{array}\right]_{l \times n} \quad b=\left[\begin{array}{c}
\beta_{1} \\
\beta_{2} \\
\vdots \\
\beta_{l}
\end{array}\right]_{l \times 1}
$$

In basic ELM, the two parameters ' $w$ ' and ' $b$ ' are selected randomly. In order to improve the classification ability, IFA is used to select these two parameters intelligently. Use classification accuracy of the training sample as fitness. Firefly will continue to search the optimal parameters by iteration.

\section{A. Simulation Verification}

In this section, two UCI data set is chosen to verify the classification ability of IFA-ELM model. In these two data sets, iris is a three classification problem, it contains 150 samples and 4 attributes; wine is a three classification problem, it contains 178 samples and 14 attributes. Considering the randomness of the data extraction, the randperm function is used to create random numbers for selecting train samples and test samples. In order to illustrate the classification performance, basic ELM model and FA-ELM model are used to compare with the IFA-ELM model. The experimental results are as follows:

TABLE I. UCI STANDARD DATA SETS

\begin{tabular}{ccc}
\hline Data sets & $\begin{array}{c}\text { Training samples’ } \\
\text { number }\end{array}$ & $\begin{array}{c}\text { Testing samples' } \\
\text { number }\end{array}$ \\
\hline Wine & 128 & 50 \\
Iris & 100 & 50 \\
\hline
\end{tabular}

TABLE II. THE COMPARISON OF CLASSIFICATION ACCURACY

\begin{tabular}{cccc}
\hline Data sets & ELM & FA-ELM & IFA-ELM \\
\hline Wine & $93.2 \%$ & $94.8 \%$ & $97.2 \%$ \\
Iris & $94 \%$ & $95.6 \%$ & $98.6 \%$ \\
\hline
\end{tabular}

For further illustrate the classification results, the experimental result figures are show as follows:
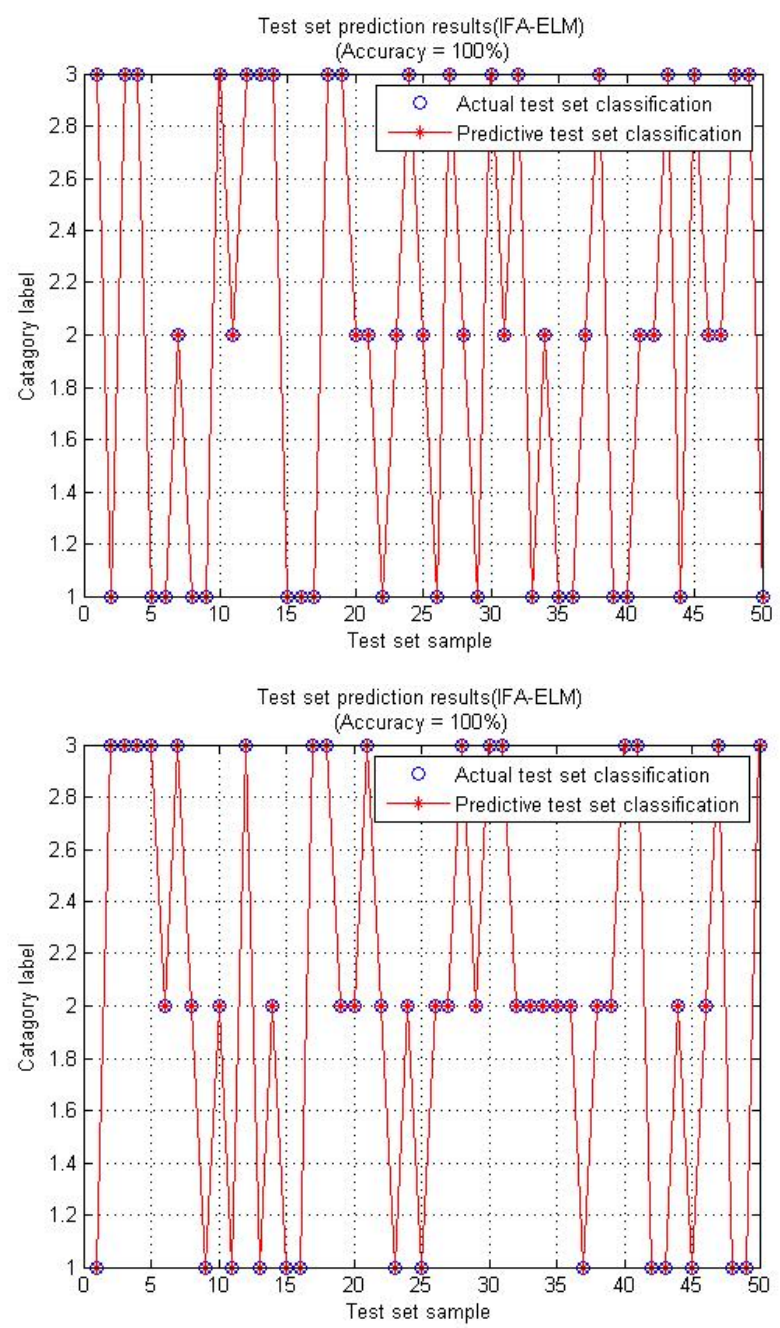

FIGURE I. IFA-ELM CLASSIFICATION RESULTS

\section{B. Application Instance}

Rolling bearing is one of the most widely used general machinery parts in various kinds of rotating machinery. 
However, $30 \%$ of the faults of rotating machinery are caused by bearing. Bearing is usually damage more frequent than other parts for its improper use [9].

Bearing's running condition can be divided into four categories: normal, ball damage, inner ring damage and out ring damage. Use IFA-ELM model to classify the fault patterns. Use '1', '2', '3', '4' to represent these four states respectively.

In this paper, we use the vibration signal collected by Bearing Data Center to do the fault diagnosis work. Then, extract characteristic values from these vibration signals. These characteristic values contain kurtosis index, skewness index, peak factor, pulse factor, margin factor, wave factor, frequency center and root mean square frequency. Part of characteristic values under different conditions are shown in the TABLE III.

Data in the Table III hasn't been normalized. In practical application, they need to be normalized to do the fault diagnosis work. Use IFA-ELM model to train the values of these samples, then put the model in the classification work of unknown samples.

In the bearing fault diagnosis experiment, we use 100 groups of experimental samples. 80 groups for training data and 20 groups for testing data. Several experiments are carried out. Considering the randomness of the data extraction, the randperm function is used to create random numbers for selecting train data and test data. The classification result figures are shown in the FIGURE II:

TABLE III. THE EXTRACTION OF CHARACTERISTIC PARAMETERS

\begin{tabular}{|c|c|c|c|c|c|c|c|c|}
\hline Practical fault type & Kurtosis index & Skewness index & Peak factor & Pulse factor & Margin factor & Wave factor & Frequency center & $\begin{array}{l}\text { Root mean square } \\
\text { frequency }\end{array}$ \\
\hline normal & 2.5637 & -0.1305 & 2.5283 & 3.1058 & 3.6373 & 1.2284 & 181.7804 & 244.7012 \\
\hline bearing boll damage & 2.4275 & 0.1101 & 3.1337 & 3.7572 & 4.3100 & 1.1990 & 633.6729 & 656.4542 \\
\hline $\begin{array}{c}\text { bearig inner ring } \\
\text { damage }\end{array}$ & 5.8305 & -0.0087 & 4.5800 & 6.4293 & 7.9738 & 1.4038 & 602.4561 & 636.5384 \\
\hline $\begin{array}{l}\text { bearing out ring } \\
\text { damage }\end{array}$ & 9.3911 & -0.0184 & 5.3392 & 8.8144 & 12.2882 & 1.6509 & 692.8669 & 701.2635 \\
\hline
\end{tabular}
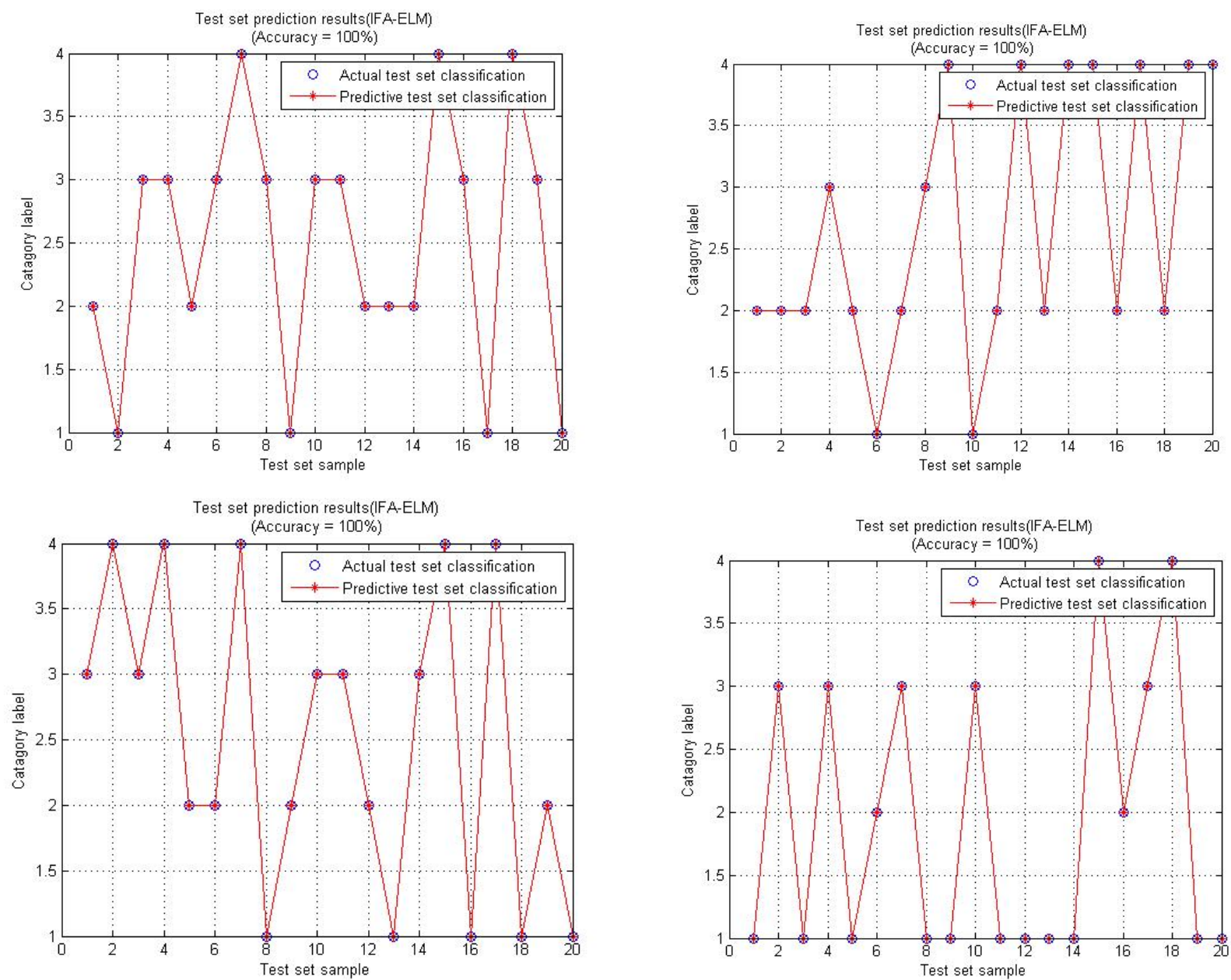

FIGURE II. IFA-ELM CLASSIFICATION RESULTS 


\section{CONCLUSION}

ELM can be used in pattern recognition. But its classification ability is not good enough. In this paper, we use improved firefly algorithm to improve its performance. IFA can solve the premature problem and we use it to select the parameters in ELM intelligently. The classification ability of IFA-ELM model can be verified by the use of UCI standard data sets. Finally, the model is used in bearing fault diagnosis work and we achieved a good result.

\section{REFERENCES}

[1] Huang G B, Zhu Q Y, Siew C K. Extreme learning machine: Theory and applications[J]. Neurocomputing, 2006, 70(1-3):489-501.

[2] Huang G B, Zhou H, Ding X, et al. Extreme learning machine for regression and multiclass classification.[J]. IEEE Transactions on Systems Man \& Cybernetics Part B Cybernetics A Publication of the IEEE Systems Man \& Cybernetics Society, 2012, 42(2):513-29.

[3] Yang X S. Firefly Algorithm, Lévy Flights and Global Optimization[M]// Research and Development in Intelligent Systems XXVI. Springer London, 2010:209-218.

[4] Senthilnath J, Omkar S N, Mani V. Clustering using firefly algorithm: Performance study[J]. Swarm \& Evolutionary Computation, 2011, 1(3):164-171.

[5] Li X, Xie H, Wang R, et al. Empirical analysis: stock market prediction via extreme learning machine[J]. Neural Computing \& Applications, 2016, 27(1):67-78.

[6] Fu Qiang, Tong Nan, Zhao Yiming. Firefly optimization algorithm based on multi group studying mechanism[j]. Computer application research, 2013, 30(12):3600-3602

[7] Wang H, Wang W, Sun H, et al. Firefly algorithm with random attraction[J]. International Journal of Bio-Inspired Computation, 2016, 8(1):33-41.

[8] Sun X, Xu J, Jiang C, et al. Extreme Learning Machine for Multi-Label Classification[J]. Entropy, 2016, 18(6).

[9] Li B, Chow M Y, Tipsuwan Y, et al. Neural-network-based motor rolling bearing fault diagnosis[J]. Industrial Electronics IEEE Transactions on, 2000, 47(5):1060-1069. 